\section{Le tissu adipeux}

\section{Un nouveau terrain de jeu pour les cellules immunitaires}

Élise Dalmas, Joan Tordjman, Michèle Guerre-Millo, Karine Clément de bas niveau dans l'obésité pouvant intervenir dans la physiopathologie de la maladie et de ses nombreuses complications est bien établie. Le tissu adipeux lui-même est un site d'inflammation où s'accumulent des macrophages. Dans cette synthèse, nous décrivons les données expérimentales et cliniques qui ont permis d'élucider certains des mécanismes cellulaires et moléculaires impliqués dans la colonisation du tissu adipeux par les macrophages obtenus à partir de précurseurs monocytaires. Les macrophages sont des cellules dont le phénotype varie suivant l'état du microenvironnement. Dans l'obésité, ils peuvent exercer des effets délétères via la production de molécules pro-inflammatoires, mais contribuent également à l'homéostasie du tissu adipeux face aux changements de la masse grasse. Une autre conséquence de l'inflammation du tissu adipeux est la présence d'une fibrose dont la genèse et les conséquences sont encore mal connues. L'identification de mécanismes potentiellement protecteurs, tels que la neutralisation immunologique de certains types de lymphocytes ou encore le contrôle transcriptionnel des gènes de l'inflammation, pourrait suggérer de nouvelles perspectives thérapeutiques pour limiter l'inflammation dans le tissu adipeux. <

En 2003, deux équipes américaines décrivent une accumulation de macrophages dans le tissu adipeux de souris obèses et chez l'homme, en relation avec l'augmentation de l'indice de masse corporelle [1, 2]. Les macrophages du tissu adipeux sont considérés comme une source majeure de facteurs pro-inflammatoires, ce qui leur confère un rôle potentiellement délétère au niveau local et systémique. Cependant, ces cellules sont particulièrement versatiles et peuvent adopter un profil anti-inflammatoire, ce qui ne permet pas d'exclure leur

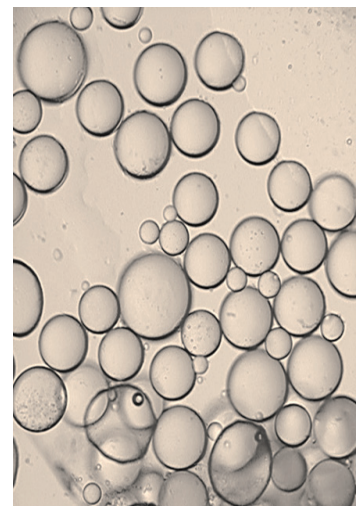

દ. Dalmas, J. Tordjman, M. Guerre-Millo : Inserm U872, équipe 7, Université Pierre et Marie Curie-Paris 6, Centre de recherche des Cordeliers, 15, rue de l'École de médecine, F-75006 Paris, France.

K. Clément : Inserm U872, équipe 7, Université Pierre et Marie Curie-Paris 6, Centre de recherche des Cordeliers; Assistance publique-Hôpitaux de Paris, Hôpital de la PitiéSalpêtrière, département de nutrition et d'endocrinologie, Paris, F-75013 France; ICAN,

Institut cardiométabolique et nutrition.

karine.clement@psl.aphp.fr adipeux a été rapportée par plusieurs

contribution physiologique à l'homéostasie du tissu adipeux. Depuis quelques années, la présence d'autres équipes, principalement dans les modèles murins d'obésité, mais également chez l'homme. Dans cette synthèse, nous montrons que ces invités inattendus, acteurs de l'immunité innée et adaptative, participent à la biologie du tissu adipeux et à ses altérations au cours du développement de l'obésité.

\section{Pourquoi le tissu adipeux est-il enflammé ?}

L'intervention de nombreux facteurs est évoquée, mais il reste difficile d'établir clairement le ou les événements à l'origine de l'inflammation dans le tissu adipeux. Les adipocytes eux-mêmes sont capables de produire de nombreuses biomolécules pro- ou anti-inflammatoires. L'accroissement de la taille des adipocytes, qui caractérise l'état obèse, altère leur profil de sécrétion et induit un statut pro-inflammatoire, alors que les signaux impliqués ne sont pas clairement définis. La répartition caractéristique des macrophages en couronne autour d'adipocytes présentant des signes de mort cellulaire [3] (Figure 1) suggère l'intervention de facteurs attractants spécifiques. Dans le tissu adipeux, des signaux exogènes comme les acides gras ou les lipopolysaccharides (LPS) d'origine bactérienne induisent une réponse inflammatoire en activant le récepteur TLR4 (toll like receptor 4 ) et les voies de signalisation intracellulaires associées, principalement la voie NFKB [4]. Localement, les adipocytes inflammatoires, dont l'activité lipolytique est élevée, libèrent des acides gras qui stimulent la voie TLR4/NFKB dans les macrophages, contribuant ainsi à 


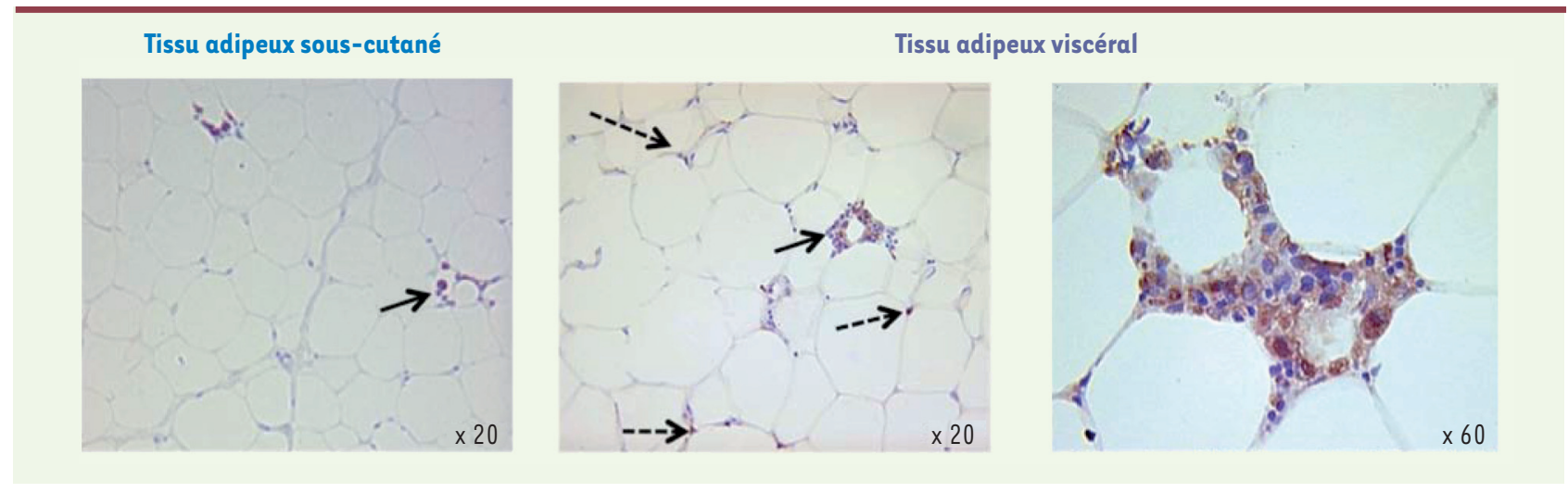

Figure 1. Aspect microscopique des macrophages dans le tissu d'un sujet obèse. Des biopsies chirurgicales de tissu adipeux sous-cutané et viscéral ont été traitées par un anticorps spécifique des macrophages. Les flèches pleines indiquent les macrophages disposés en couronne autour d'un adipocyte ; les flèches pointillées indiquent les macrophages disposés à l'intersection des adipocytes dans le parenchyme.

une boucle paracrine délétère entre les deux types cellulaires [5]. Dans ce contexte, l'inhibition de TLR4 par des facteurs tels que ATF3, facteur de transcription de la famille ATF/CREB, ou CTRP-3 (Clq/TNF[tumor necrosis factor]-related protein-3), membre de la famille $\mathrm{Clq} / \mathrm{TNF}$, pourrait représenter une cible thérapeutique pour contrôler l'inflammation du tissu adipeux $[5,6]$. Récemment, un rôle anti-inflammatoire des acides gras omega 3 agissant via le récepteur GPR120 des macrophages a été identifié ; cette nouvelle approche reste à explorer [7]. Parmi d'autres facteurs, I'hypoxie tissulaire, cause classique d'attraction des macrophages dans certaines tumeurs et dans la plaque d'athérome, pourrait aussi intervenir dans le déclenchement de l'inflammation du tissu adipeux chez les sujets obèses [8]. Le stress du réticulum endoplasmique [44] et le stress oxydant [45] seraient aussi susceptibles d'intervenir dans l'inflammation locale. Les interactions entre ces différents types de stress cellulaire et les biomolécules de l'inflammation ouvrent tout un champ d'explorations au cours de l'évolution de l'obésité.

\section{Lutte d'influence entre les cellules immunitaires dans le tissu adipeux}

L'inflammation intervient dans le tissu adipeux alors qu'aucun microorganisme pathogène n'a été clairement identifié à ce jour, ce qui permet de la qualifier d'inflammation «stérile ». Plusieurs observations chez la souris rendue obèse par un régime hyperlipidique (DIO, diet-induced obesity) sont en faveur de l'intervention d'antigènes non microbiens. Ainsi, l'infiltration précoce et transitoire de neutrophiles suggère qu'une inflammation « aiguë » précède l'apparition des macrophages dans ce modèle [9]. Des cellules NKT (natural killer T), capables de reconnaître des antigènes glycolipidiques, colonisent également le tissu adipeux dans les premières phases de l'obésité, étape qui semble nécessaire à l'infiltration macrophagique [10]. Dans un modèle murin (souris $\left.K i t^{W-s h / W-s h}\right)^{1}$, l'absence de mastocytes réduit

${ }^{1}$ Ces souris sont dépourvues du récepteur c-Kit, dont le ligand physiologique est le SCF (stem cell factor), cytokine essentielle à la différenciation des mastocytes. l'accumulation de macrophages dans le tissu adipeux. Comment ces cellules surtout impliquées dans l'allergie contribuent au processus inflammatoire du tissu adipeux reste à clarifier [11]. Des lymphocytes, particulièrement des lymphocytes $T$, ont également été détectés dans le tissu adipeux chez la souris et chez l'homme (Tableau 1). Des études cinétiques dans le modèle DIO révèlent que les lymphocytes $T$ précèdent les macrophages dans le tissu adipeux $[12,13]$. De plus, les lymphocytes $T \mathrm{CD} 4^{+}$isolés du tissu adipeux de souris DIO expriment un répertoire TCR restreint, suggérant qu'ils puissent reconnaître un - ou des - antigènes d'origine adipeuse [14].

On distingue plusieurs profils de mobilisation des lymphocytes $T$ dans le tissu adipeux dans l'obésité : le nombre de lymphocytes $T$ cytotoxiques $\left(C D 8^{+}\right)$augmente alors que celui des lymphocytes T anti-inflammatoires Th2 $\left(\mathrm{CD}^{+} \mathrm{GATA}^{+}\right)$et Treg $\left(\mathrm{Foxp}^{+}\right)$diminue [13-15]. L'importance physiopathologique de ces changements est illustrée par le fait que l'inactivation des T CD8 $8^{+}$[13] ou l'induction des lymphocytes Th2 ou des Treg $[14,15]$ diminue l'inflammation dans le tissu adipeux des souris DIO. Chez l'homme, une étude montre l'augmentation des lymphocytes T CD8 ${ }^{+}$et lymphocytes $\mathrm{CD}^{+}$avec le degré d'adiposité dans le tissu adipeux [16].

L'ensemble de ces observations suggèrent l'existence d'un jeu complexe d'influence entre les différents acteurs cellulaires de l'immunité innée (neutrophiles, mastocytes), adaptative (lymphocytes) ou à l'interface des deux systèmes (NKT). Ces différents protagonistes semblent agir séquentiellement ou de concert pour favoriser in fine l'accumulation de macrophages dans le tissu adipeux par des mécanismes encore mal connus. Un enjeu majeur de ces études reste l'identification 


\begin{tabular}{|c|c|c|c|c|c|}
\hline \multirow{2}{*}{$\begin{array}{l}\text { Système } \\
\text { immunitaire }\end{array}$} & \multicolumn{2}{|c|}{ Cellules de l'immunité } & \multirow{2}{*}{ Marqueurs $^{1}$} & \multirow{2}{*}{$\begin{array}{l}\text { Effet de } \\
\text { l'obésité }\end{array}$} & \multirow{2}{*}{ Références } \\
\hline & Type cellulaire & Phénotype & & & \\
\hline \multirow{6}{*}{ Inné } & Macrophages & & $\mathrm{CD} 68, \mathrm{CD} 14, \mathrm{~F} 4 / 80$ & $\uparrow$ & {$[1-3,23,38,40]$} \\
\hline & & $\mathrm{Ml}^{2}$ & $\begin{array}{l}\text { CD40, CD1lc, CD } 86 \text {, } \\
\text { Mincle }\end{array}$ & $\uparrow$ & {$[17,27,32,33,35,36]$} \\
\hline & & $M 2^{2}$ & CD206, CD163, CD209 & $\uparrow \leftrightarrow$ & {$[17,27,32]$} \\
\hline & & Mixte M1 M2 & & $\uparrow$ & [29-31] \\
\hline & Neutrophiles & & NIMP-R-14 & $\uparrow$ & [9] \\
\hline & Mastocytes & & Tryptase, chymase & $\uparrow$ & {$[11]$} \\
\hline Interface & NKT & & CD3 et CD56, NK1.1 & $\uparrow$ & {$[10]$} \\
\hline \multirow{6}{*}{ Adaptatif } & Lymphocytes T & & $\mathrm{CD} 3$ & $\uparrow$ & {$[13-16]$} \\
\hline & & Cytotoxiques & $\mathrm{CD} 3$ et $\mathrm{CD} 8$ & $\uparrow$ & {$[13,14,16]$} \\
\hline & & Auxiliaires & $\mathrm{CD} 3$ et $\mathrm{CD} 4$ & $\downarrow \uparrow$ & {$[13,14,16]$} \\
\hline & & $\mathrm{T}_{\mathrm{H}} \mathrm{l}$ & CD3 et CD4 et IFN $\gamma$ & $\uparrow$ & [14] \\
\hline & & $\mathrm{T}_{\mathrm{H}} 2$ & CD3 et CD4 et Gata3 & $\leftrightarrow$ & [14] \\
\hline & & $\mathrm{T}_{\text {reg }}$ & $\begin{array}{l}\text { CD3 et CD4 et Foxp3, } \\
\text { CD25 }\end{array}$ & $\downarrow$ & {$[13-15]$} \\
\hline
\end{tabular}

Tableau I. Les cellules de l'immunité dans le tissu adipeux. 'Sélection de marqueurs couramment utilisés. ${ }^{2}$ Marqueurs d'activation MI ou M2 associés aux marqueurs de macrophages, CD68 ou CD14 chez l'homme et F4/80 chez les rongeurs.

du ou des facteurs capables de déclencher une réponse immunitaire dans le tissu adipeux au cours des phases d'évolution de l'obésité humaine.

\section{D’où viennent les macrophages du tissu adipeux ?}

Les études de transplantation de moelle osseuse chez la souris ont clairement montré que les monocytes circulants sont les précurseurs de la majorité des macrophages présents dans le tissu adipeux [2], ce qui a été confirmé par des expériences de marquage des monocytes in vivo [17]. Plusieurs sous-populations de monocytes ont été décrites chez la souris et chez l'homme. Leur devenir dépend de leur phénotype, d'une part, et du microenvironnement dans lequel elles vont se différencier en macrophages, d'autre part. Chez la souris, les monocytes Grl $1^{+}$Ly- $6 C^{\text {high }}$, équivalents des monocytes classiques $C D 14^{+} C D 16^{-}$chez I'homme, seraient les précurseurs des macrophages pro-inflammatoires du tissu adipeux $[18,19]$. Chez l'homme, des premiers travaux ont révélé que les cellules mononucléées circulantes expriment un statut pro-inflammatoire chez les sujets obèses [20]. Plus récemment, une étude clinique indique que la fréquence d'une sous-population de monocytes $C D 14^{\text {dim }} C D 16^{+}$récemment identifiée est augmentée chez des sujets en surpoids [21]. Cette souspopulation monocytaire pourrait contribuer préférentiellement à l'accumulation des macrophages dans le tissu adipeux.

Les mécanismes cellulaires et moléculaires qui favorisent le recrutement des monocytes via leur diapédèse à travers l'endothélium vasculaire sont peu explorés dans le tissu adipeux. Plusieurs molécules chémoattractantes peuvent intervenir, et un rôle important du système MCP-1/CCR2 (monocyte chemotactic protein 1 et son récepteur (CR2) dans l'accumulation des macrophages du tissu adipeux a été suggéré par l'étude de modèles murins [22]. Les adipocytes eux-mêmes produisent des facteurs solubles qui augmentent la diapédèse monocytaire et stimulent la production de protéines d'adhésion, dont PECAM-1 (platelet endothelial cell adhesion molecule) et ICAM-1 (intercellular adhesion molecule) exprimées sur les cellules endothéliales [23]. Nos travaux montrent que la cytokine CCL5 (alias RAN- 


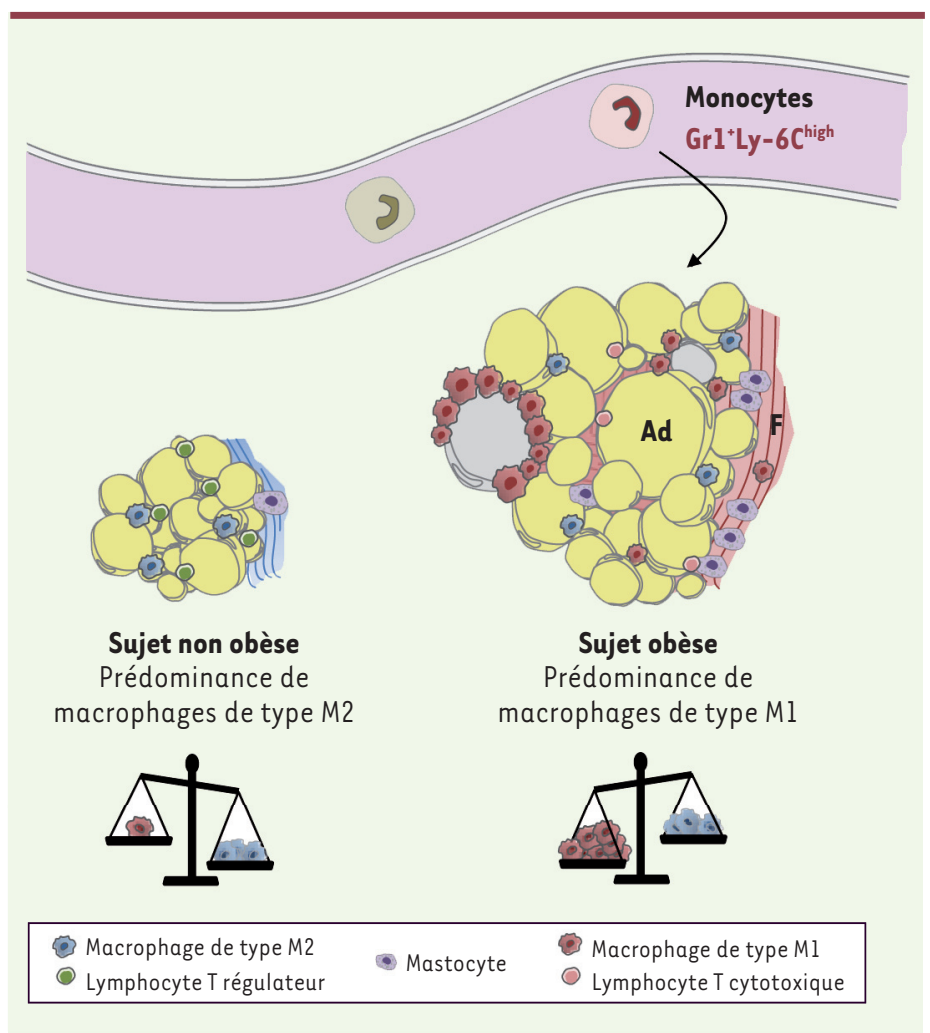

Figure 2. Altérations cellulaires et structurales du tissu adipeux induites par l'obésité. Chez les souris non obèses, les macrophages résidents ont un phénotype de type M2. Des lymphocytes Treg sont présents, particulièrement dans les tissus profonds. Dans le modèle DIO, I'obésité induit le recrutement de monocytes inflammatoires de phénotype $\mathrm{Gr} \mathrm{l}^{+} \mathrm{L} y-6 \mathrm{C}^{\text {high }}$, qui seraient les précurseurs de macrophages de type Ml. Ces macrophages recrutés sont disposés en couronne autour d'adipocytes présentant des caractéristiques de mort cellulaire. Les mécanismes impliqués sont divers et complexes et pourraient mettre en jeu la contribution d'autres cellules de l'immunité, dont les lymphocytes cytotoxiques. La présence d'une fibrose a été décrite principalement dans le tissu adipeux humain (voir Figure 3). Plusieurs types cellulaires sont associés aux zones fibrotiques, dont des macrophages et des mastocytes. Ad : adipocytes ; $\mathrm{F}$ : fibrose.

TES [regulated upon activation, normal T cell expressed and secreted]) pourrait contribuer à ce processus [24]. II s'avère que les cellules endothéliales sont plus inflammatoires dans le tissu adipeux viscéral que dans le tissu adipeux superficiel, ce qui pourrait favoriser le recrutement des monocytes dans les tissus profonds [25]. Clairement, d'autres études sont nécessaires pour définir le spectre complet des facteurs contribuant à la diapédèse des monocytes et à l'activation endothéliale dans le tissu adipeux au cours de l'obésité.

\section{Les macrophages du tissu adipeux : un phénotype variable et complexe}

Il existe un large spectre phénotypique de macrophages, mais on distingue surtout deux catégories suivant une classification qui s'apparente à celle des lymphocytes Th1/Th2 : les macrophages Ml activés par la voie classique et les macrophages M2 qui utilisent une voie alterne d'activation [46]. Comme pour d'autres types cellulaires, cette classification repose en partie sur l'expression de marqueurs dont la spécificité reste souvent imprécise (Tableau I). Les macrophages Ml sont stimulés par le LPS, l'interféron- $\gamma$ et le TNF- $\alpha$ et produisent des cytokines pro-inflammatoires comme l'IL(interleukine)- 6 et l'IL- 1 . Les macrophages M2 sont induits par I'IL-4 et IL-13 et, via la production de facteurs tels que l'IL-10 et le TGF- $\beta$ (transforming growth factor), ont un effet anti-inflammatoire. À ces phénotypes sont associées des fonctions distinctes : élimination des pathogènes et induction d'inflammation pour les macrophages $\mathrm{Ml}$, angiogenèse et réparation tissulaire pour les macrophages M2. Qu'en est-il dans le tissu adipeux ? L'injection d'un marqueur spécifique des monocytes chez la souris a permis de montrer que l'obésité (modèle DIO) provoque le recrutement de macrophages $\mathrm{Ml}$, qui sont ceux qui s'organisent en couronne autour des adipocytes [26]. Ces macrophages sont chargés en gouttelettes lipidiques, qui témoignent de leur activité de phagocytose des lipides [1, 3]. En l'absence d'obésité, des macrophages résidents sont également détectés dans le tissu adipeux. Ils présentent un phénotype de type M2 et leur nombre n'est pas ou peu modifié dans l'obésité [26, 27]. Une étude cinétique dans le modèle DIO suggère une situation plus complexe : l'accumulation de macrophages de type $\mathrm{Ml}$ se poursuit jusqu'à 16 semaines de régime puis diminue au profit de macrophages de type M2, sans que l'on sache s'il s'agit d'un recrutement ou d'une repolarisation de ces macrophages in situ [28]. De plus, une troisième population de phénotype intermédiaire $\mathrm{Ml} / \mathrm{M} 2$ a été identifiée dans le tissu adipeux de souris DIO ; elle pourrait constituer un groupe de cellules en cours de repolarisation [29].

Dans le tissu adipeux humain, les macrophages sont essentiellement de phénotype mixte intermédiaire entre les états de polarisation Ml et M2 [30,31]. Dans une population de sujets massivement obèses, nous avons observé une augmentation du nombre des cellules positives pour le marqueur CD40 et un nombre constant de cellules exprimant des marqueurs de type M2 (CD206 [mannose receptor] et CD163 [membre de la famille des scavenger receptors]) par rapport à des sujets témoins, suggérant que l'obésité favorise l'accumulation de macrophages de type Ml [32]. Une caractérisation plus 
précise montre que, comme chez la souris, les macrophages disposés en couronne autour des adipocytes présentent un phénotype de type $\mathrm{Ml}$, alors que les macrophages interstitiels ont un phénotype orienté M2 [33, 34]. Chez l'homme, cependant, il est impossible de distinguer les macrophages recrutés des macrophages résidents.

Les lipides activateurs de la voie TLR4/NFKB [35] ou l'accumulation de lipides dans les macrophages eux-mêmes [36] favorisent la polarisation MI dans le tissu adipeux des souris DIO. De manière surprenante, l'augmentation de la capacité de stockage des triglycérides dans les macrophages via la surexpression de la diacylglycérol acyltransférase l (DGATl) les rend résistants à une activation Ml [37]. Ce phénotype s'explique par le fait que les triglycérides seraient moins pro-inflammatoires que certaines autres espèces lipidiques dans les macrophages. L'ensemble de ces travaux chez la souris et chez l'homme révèlent la complexité et l'aspect évolutif du phénotype des macrophages du tissu adipeux dans l'obésité (Figure 2), une situation certainement associée à des aspects fonctionnels qui restent à clarifier.

\section{Les macrophages du tissu adipeux : amis ou ennemis?}

La question du rôle des macrophages dans les complications métaboliques de l'obésité s'est rapidement posée. Dans les modèles murins, l'abondance des macrophages peut être modifiée dans le tissu adipeux par des moyens pharmacologiques ou via des manipulations génétiques ciblées, ce qui a permis d'établir l'importance de ces cellules dans l'induction d'une insulinorésistance. Chez l'homme, en revanche, cette relation reste plutôt controversée. Certaines études rapportent une relation inverse entre la sensibilité à l'insuline et l'expression de marqueurs de macrophages dans le tissu adipeux, particulièrement dans le tissu adipeux viscéral [38]. La présence accrue de macrophages en couronne a également été associée à l'altération de la sensibilité à l'insuline [39]. À l'inverse, nos travaux ne permettent pas d'établir de relation entre l'abondance des macrophages et les paramètres sanguins de l'homéostasie glucidique chez des sujets très obèses [40]. L'association entre l'abondance d'une fraction minoritaire de macrophages de phénotype mixte et l'insulinorésistance a été rapportée, suggérant qu'une analyse précise des phénotypes pourrait permettre de distinguer des types de macrophages plus délétères que d'autres [33].

Bien que fortement soupçonné, un lien entre l'accumulation macrophagique et l'augmentation du risque cardiovasculaire n'a pu être établi chez l'homme. En revanche, nos études cliniques dans une large population de sujets massivement obèses révèlent que la gravité de l'histopathologie hépatique (stéatose et fibrose inflammatoire) est liée à l'ampleur de l'accumulation macrophagique dans le tissu adipeux viscéral, indépendamment du statut glycémique [40]. Les facteurs qui relaient les signaux pro-inflammatoires du tissu adipeux viscéral vers le foie sont en cours d'identification.

Les macrophages peuvent-ils contribuer à maintenir l'homéostasie du tissu adipeux face aux changements du bilan énergétique ? Une

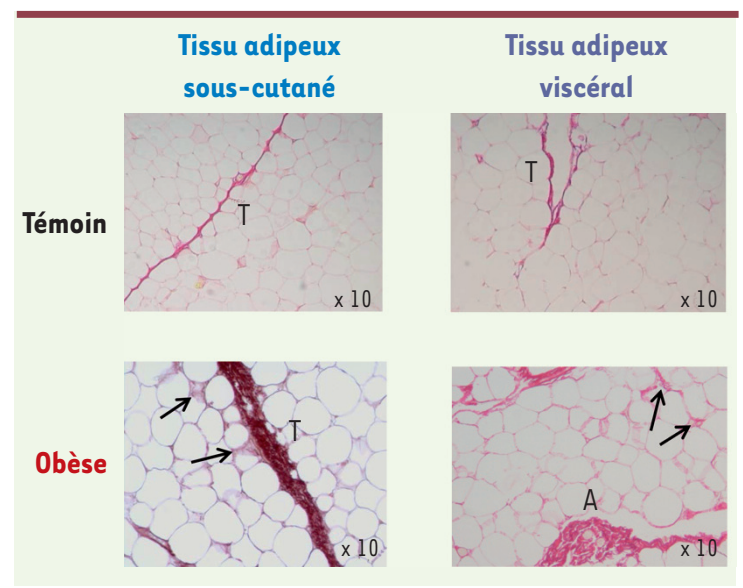

Figure 3. Aspect microscopique des dépôts de fibrose dans le tissu adipeux humain. Des biopsies chirurgicales de tissu adipeux sous-cutané et viscéral ont été marquées au rouge picrosirius (marqueur des collagènes) pour révéler les zones fibrotiques. A : amas; T : travée. Les flèches localisent la fibrose péricellulaire présente uniquement chez les sujets obèses.

étude cinétique au cours de la perte de poids chez la souris DIO permet de répondre en partie à cette question. En effet, contrairement à l'hypothèse initiale, une accumulation importante de macrophages a été observée dans le tissu adipeux lors des phases précoces d'une restriction calorique, au moment où la lipolyse adipocytaire est stimulée. Ces macrophages exercent une activité de phagocytose des lipides sans déclencher d'inflammation, et contribuent ainsi à restaurer localement I'homéostasie lipidique [41]. D'autres observations chez la souris suggèrent que la présence de macrophages pourrait contribuer à contenir l'hypertrophie adipocytaire. Cette possibilité est évoquée pour rendre compte du phénotype des souris déficientes pour le gène CCR2, qui code pour le récepteur de MCP1 : elles présentent une diminution du nombre des macrophages associée à une augmentation du diamètre adipocytaire [26]. II faut noter que les adipocytes étant les seules cellules de l'organisme capables de stocker efficacement et sans dommage l'excès d'apport calorique sous forme de triglycérides, la limitation de leur expansion peut se révéler plus néfaste que bénéfique en favorisant les dépôts ectopiques dans d'autres organes non spécialisés tels que le foie.

\section{La fibrose, une nouvelle conséquence de l'inflammation du tissu adipeux}

Associé à une inflammation chronique, le remodelage de la matrice extracellulaire peut aboutir à une accumulation anormale d'éléments matriciels qui carac- 
térise la fibrose. C'est effectivement ce qui se produit dans le tissu adipeux humain, où nous avons détecté récemment la présence de dépôts fibrotiques en amas denses ou en travées [42]. Chez le sujet obèse, il existe également de la fibrose péricellulaire entourant les adipocytes (Figure 3). Les macrophages sont considérés comme des régulateurs majeurs de la fibrose dans différents tissus où ils exercent des rôles pro- ou antifibrotiques suivant leur phénotype. In vitro, le milieu conditionné de macrophages Ml activés par le LPS ou isolés du tissu adipeux de sujets obèses confère un phénotype profibrotique aux préadipocytes humains [43]. Ces observations suggèrent un rôle plutôt profibrotique des macrophages du tissu adipeux chez les sujets obèses.

Quelles sont les conséquences de la présence de fibrose dans le tissu adipeux ? Dans notre étude chez des sujets obèses, nous observons que plus la quantité de fibrose est faible, plus les sujets perdent de la masse grasse en réponse à la chirurgie gastrique. La présence de fibrose en excès pourrait altérer le remodelage du tissu adipeux et potentiellement les adaptations métaboliques qui interviennent au cours de la perte de poids rapide et drastique induite dans ce modèle [42]. Élucider les acteurs cellulaires et moléculaires de la fibrose et ses conséquences locales ou systémiques est un nouveau défi dans le domaine de l'obésité.

\section{Conclusion}

Dans l'obésité, le tissu adipeux blanc est la cible d'un remaniement cellulaire et structural majeur dont les composants ne sont probablement pas encore tous identifiés. Les types cellulaires et les signaux impliqués sont multiples et complexes, créant localement un microenvironnement pro-inflammatoire qui favorise le recrutement de macrophages et la formation de fibrose. La cinétique de ces altérations est également mal connue au cours des différentes phases du développement de l'obésité. Bien que partiellement réversibles, certaines de ces altérations persistent après une perte de poids. L'identification des mécanismes potentiellement protecteurs, tels que la neutralisation immunologique de certains types de lymphocytes ou encore le contrôle transcriptionnel de l'inflammation, pourrait ouvrir de nouvelles perspectives thérapeutiques visant à contenir l'inflammation dans le tissu adipeux. $\diamond$

\section{SUMMARY}

Adipose tissue, a new playground for immune cells

Adipose tissue has been under focus in the last decade and pivotal concepts have emerged from the studies of its complex biology. Low-grade inflammation both at the systemic level and in adipose tissue itself characterizes obesity. Among the different cell types contributing to inflammation, this review focuses on the mechanisms and consequences of macrophage accumulation in obese adipose tissue. Mechanisms for monocyte recruitment to adipose tissue, and how macrophages' phenotypes are modified in this environment in response to increasing fat mass, are considered. We review recent studies addressing the complex and versatile phenotype of adipose tissue macrophages that contribute to inflammatory and metabolic alterations, but could also help to maintain adipose tissue homeostasis in the setting of obesity both in mouse and human situations. A newly discovered consequence of adipose tissue inflammation is fibrosis. Whether macrophages and/or other immune cells exert a pro-fibrotic effect in adipose tissue is still unclear. This wealth of new information will hopefully help to design new ways to control adipose tissue inflammation and its deleterious sequels. $\diamond$

\section{CONFLIT D'INTÉRÊTS}

Les auteurs déclarent n'avoir aucun conflit d'intérêts concernant les données publiées dans cet article.

\section{REMERCIEMENTS}

Les auteurs remercient la Commission Européenne ( $6^{\text {th }}$ framework program Hepadip; $7^{\text {th }}$ framework programs ADAPT, FLIP) et la Région île-de-France (CODDIM) qui soutiennent financièrement leur recherche dans le domaine de l'obésité et de l'inflammation.

\section{RéFÉRENCES}

1. Xu H, Barnes GT, Yang $Q$, et al. Chronic inflammation in fat plays a crucial role in the development of obesity-related insulin resistance. J Clin Invest $2003 ; 112: 1821-30$

2. Weisberg SP, McCann D, Desai M, et al. Obesity is associated with macrophage accumulation in adipose tissue. J Clin Invest 2003; 112 : 1796808.

3. Cinti S, Mitchell G, Barbatelli G, et al. Adipocyte death defines macrophage localization and function in adipose tissue of obese mice and humans. J Lipid Res $2005 ; 46: 2347-55$.

4. Shi H, Kokoeva MV, Inouye K, et al. TLR4 links innate immunity and fatty acid-induced insulin resistance. J Clin Invest 2006 ; 116 : 3015-25.

5. Suganami T, Yuan X, Shimoda Y, et al. Activating transcription factor 3 constitutes a negative feedback mechanism that attenuates saturated fatty acid/toll-like receptor 4 signaling and macrophage activation in obese adipose tissue. Circ Res 2009 ; $105: 25-32$.

6. Kopp A, Bala M, Buechler C, et al. Clq/TNF-related protein-3 represents a novel and endogenous lipopolysaccharide antagonist of the adipose tissue. Endocrinology $2010 ; 151: 5267-78$.

7. Oh da Y, Talukdar S, Bae $\varepsilon$ J, et al. GPR120 is an omega-3 fatty acid receptor mediating potent anti-inflammatory and insulin-sensitizing effects. Cell $2010 ; 142: 687-98$.

8. Ye J. Emerging role of adipose tissue hypoxia in obesity and insulin resistance. Int J Obes (Lond) $2009 ; 33: 54-66$.

9. Elgazar-Carmon V, Rudich A, Hadad N, Levy R. Neutrophils transiently infiltrate intra-abdominal fat early in the course of high-fat feeding. J Lipid Res 2008 ; 49 : 1894-903.

10. Ohmura K, Ishimori N, Ohmura Y, et al. Natural killer T cells are involved in adipose tissues inflammation and glucose intolerance in diet-induced obese mice. Arterioscler Thromb Vasc Biol 2010 ; 30 : 193-9.

11. Liu J, Divoux A, Sun J, et al. Genetic deficiency and pharmacological stabilization of mast cells reduce diet-induced obesity and diabetes in mice. Nat Med 2009 ; 15 : 940-5.

12. Kintscher $U$, Hartge $M$, Hess $K$, et al. T-lymphocyte infiltration in visceral adipose tissue: a primary event in adipose tissue inflammation and the development of obesity-mediated insulin resistance. Arterioscler Thromb Vasc Biol 2008 ; 28 : 1304-10.

13. Nishimura S, Manabe I, Nagasaki M, et al. CD8 ${ }^{+}$effector T cells contribute to macrophage recruitment and adipose tissue inflammation in obesity. Nat Med 2009; 15 : 914-20. 
14. Winer $S$, Chan $Y$, Paltser $G$, et al. Normalization of obesity-associated insulin resistance through immunotherapy. Nat Med 2009 ; 15 : $921-9$.

15. Feuerer M, Herrero L, Cipolletta D, et al. Lean, but not obese, fat is enriched for a unique population of regulatory T cells that affect metabolic parameters. Nat Med $2009 ; 15: 930-9$.

16. Duffaut C, Zakaroff-Girard A, Bourlier V, et al. Interplay between human adipocytes and T lymphocytes in obesity: CCL2O as an adipochemokine and T lymphocytes as lipogenic modulators. Arterioscler Thromb Vasc Biol 2009; 29 : 1608-14.

17. Lumeng CN, Deyoung SM, Bodzin JL, Saltiel AR. Increased inflammatory properties of adipose tissue macrophages recruited during diet-induced obesity. Diabetes $2007 ; 56: 16-23$.

18. Tsou CL, Peters W, Si Y, et al. Critical roles for CCR2 and MCP-3 in monocyte mobilization from bone marrow and recruitment to inflammatory sites. J Clin Invest 2007 ; 117 : 902-9.

19. Westcott DJ, Delproposto JB, Geletka LM, et al. MGLI promotes adipose tissue inflammation and insulin resistance by regulating 7/4hi monocytes in obesity. J Exp Med 2009; 206 : 3143-56.

20. Ghanim H, Aljada A, Hofmeyer D, et al. Circulating mononuclear cells in the obese are in a proinflammatory state. Circulation $2004 ; 110: 1564-71$.

21. Rogacev KS, Ulrich C, Blomer L, et al. Monocyte heterogeneity in obesity and subclinical atherosclerosis. Eur Heart J $2010 ; 31: 369-76$.

22. Sell H, Eckel J. Monocyte chemotactic protein-l and its role in insulin resistance. Curr Opin Lipidol $2007 ; 18: 258-62$.

23. Curat CA, Miranville A, Sengenes C, et al. From blood monocytes to adipose tissue-resident macrophages: induction of diapedesis by human mature adipocytes. Diabetes $2004 ; 53$ : 1285-92.

24. Keophiphath M, Rouault C, Divoux A, et al. CCL5 promotes macrophage recruitment and survival in human adipose tissue. Arterioscler Thromb Vasc Biol $2010 ; 30: 39-45$.

25. Villaret A, Galitzky J, Decaunes P, et al. Adipose tissue endothelial cells from obese human subjects: differences among depots in angiogenic, metabolic, and inflammatory gene expression and cellular senescence. Diabetes $2010 ; 59: 2755-63$.

26. Lumeng CN, Bodzin JL, Saltiel AR. Obesity induces a phenotypic switch in adipose tissue macrophage polarization. J Clin Invest 2007 ; 117 : 175-84.

27. Fujisaka S, Usui I, Bukhari A, et al. Regulatory mechanisms for adipose tissue $\mathrm{Ml}$ and $\mathrm{M} 2$ macrophages in diet-induced obese mice. Diabetes $2009 ; 58: 2574-82$.

28. Strissel KJ, Stancheva Z, Miyoshi H, et al. Adipocyte death, adipose tissue remodeling, and obesity complications. Diabetes $2007 ; 56: 2910-8$.

29. Shaul ME, Bennett G, Strissel KJ, et al. Dynamic, M2-like remodeling phenotypes of CD1l lc adipose tissue macrophages during high-fat diet : induced obesity in mice. Diabetes $2010 ; 59: 1171-81$.

30. Zeyda M, Farmer D, Todoric J, et al. Human adipose tissue macrophages are of an antiinflammatory phenotype but capable of excessive pro-inflammatory mediator production. Int J Obes (Lond) $2007 ; 31: 1420-8$.

31. Bourlier V, Zakaroff-Girard A, Miranville A, et al. Remodeling phenotype of human subcutaneous adipose tissue macrophages. Circulation 2008 ; 117 : 806-15.

32. Aron-Wisnewsky J, Tordjman J, Poitou C, et al. Human adipose tissue macrophages: $\mathrm{ml}$ and $\mathrm{m} 2$ cell surface markers in subcutaneous and omental depots and after weight loss. J Clin Endocrinol Metab $2009 ; 94: 4619-23$.
33. Wentworth JM, Naselli G, Brown WA, et al. Pro-inflammatory CD11 $\mathrm{c}^{+} \mathrm{CD} 206^{+}$ adipose tissue macrophages are associated with insulin resistance in human obesity. Diabetes $2010 ; 59: 1648-56$

34. Spencer M, Yao-Borengasser A, Unal R, et al. Adipose tissue macrophages in insulin resistant subjects are associated with collagen $\mathrm{VI}$, fibrosis and demonstrate alternative activation. Am J Physiol Endocrinol Metab 2010 ; 299 : ع1016-27.

35. Ichioka M, Suganami T, Tsuda N, et al. Increased expression of macrophageinducible $\mathrm{C}$-type lectin in adipose tissue of obese mice and humans. Diabetes $2011 ; 60: 819-26$

36. Prieur X, Mok CY, Velagapudi VR, et al. Differential lipid partitioning between adipocytes and tissue macrophages modulates macrophage lipotoxicity and $\mathrm{m} 2 / \mathrm{ml}$ polarization in obese mice. Diabetes $2011 ; 60: 797-809$.

37. Koliwad SK, Streeper RS, Monetti M, et al. DGATl-dependent triacylglycerol storage by macrophages protects mice from diet-induced insulin resistance and inflammation.J Clin Invest 2010 ; $120: 756-67$.

38. Harman-Boehm I, Bluher $\mathrm{M}$, Redel $\mathrm{H}$, et al. Macrophage infiltration into omental versus subcutaneous fat across different populations: effect of regional adiposity and the comorbidities of obesity. J Clin Endocrinol Metab $2007 ; 92: 2240-7$

39. Apovian CM, Bigornia S, Mott M, et al. Adipose macrophage infiltration is associated with insulin resistance and vascular endothelial dysfunction in obese subjects. Arterioscler Thromb Vasc Biol 2008 ; 28 : 1654-9.

40. Tordjman J, Poitou C, Hugol D, et al. Association between omental adipose tissue macrophages and liver histopathology in morbid obesity: influence of glycemic status. J Hepatol 2009 ; $51: 354-62$.

41. Kosteli A, Sugaru $\varepsilon$, Haemmerle G, et al. Weight loss and lipolysis promote a dynamic immune response in murine adipose tissue. J Clin Invest 2010; 120 : 3466-79.

42. Divoux A, Tordjman J, Lacasa D, et al. Fibrosis in human adipose tissue: composition, distribution, and link with lipid metabolism and fat mass loss. Diabetes $2010 ; 59$ : 2817-25.

43. Keophiphath M, Achard V, Henegar C, et al. Macrophage-secreted factors promote a profibrotic phenotype in human preadipocytes. Mol Endocrinol $2009 ; 23: 11-24$.

44. Foufelle F, Ferré P. La réponse UPR : son rôle physiologique et physiopathologique. Med Sci (Paris) 2007 ; $23: 291-6$.

45. Migdal C, Serres M. Espèces réactives de l'oxygène et stress oxydant. Med Sci (Paris) 2011; $27:$ 405-12.

46. Mege JL, Capo C. La polarisation des macrophages, le nœud gordien des infections bactériennes? Med Sci (Paris) $2010 ; 26: 83-8$.

TIRÉS À PART

K. Clément

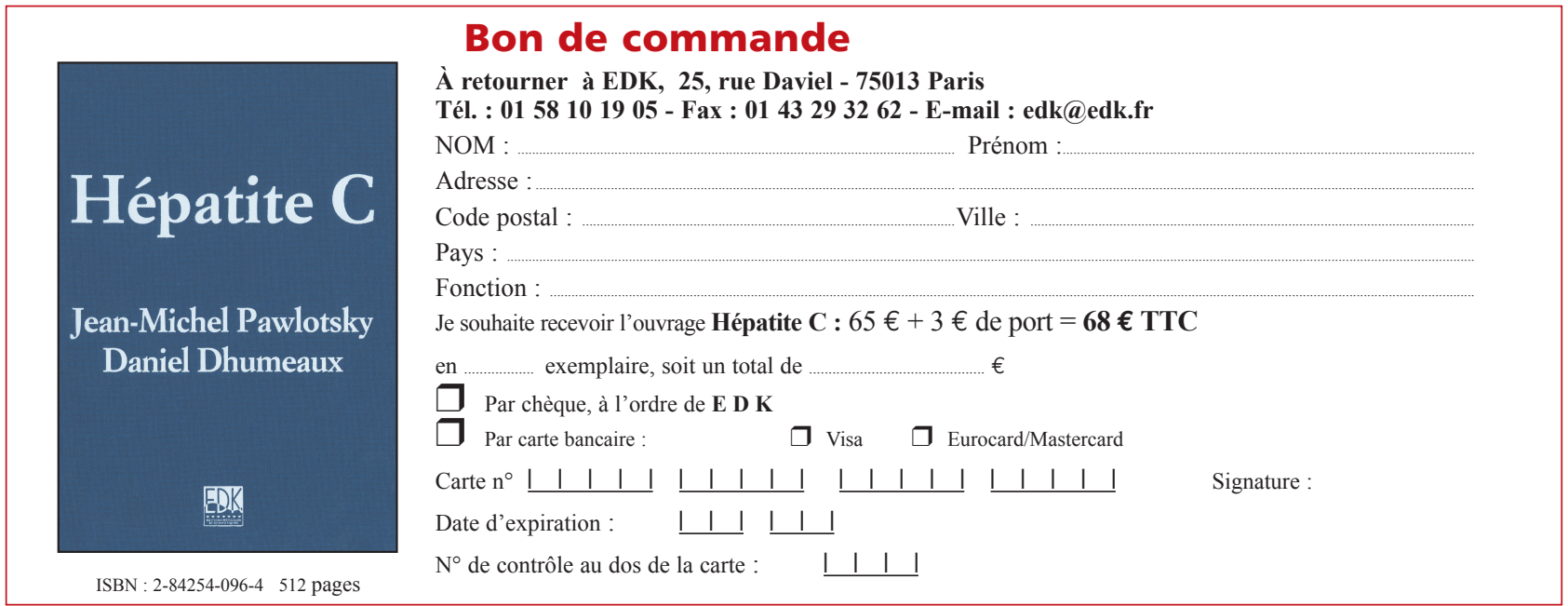

